\title{
Capability of Electron Microscopy in Forensic Science
}

\author{
Marek Kotrlý1,2 \\ ${ }^{1}$ Institute of Criminalistics Prague (ICP), Czech Republic; \\ ${ }^{2}$ Charles University in Prague, Faculty of Science, Praha, Czech Republic.
}

Electron microscopy and integrated microanalysis (EDS / WDS / XRF) are among the basic applications that are used to investigate traces from crime scene and comparisons in forensic practice. These techniques allow both rapid screening and basic information for a wide range of traces, e.g. unknown samples, as well as detailed identification and comparison across a variety of forensic disciplines. The materials analyzed by electron microscopy are very diverse, practically any material that originates from the activities of the human being and the nature and which has some connection with the case (from the fragment of the prehistoric vessel through the data records, documents, materials for high-tech semiconductors) can be found in the forensic laboratory. Therefore, materials of organic origin, fragments of plants and animals, etc. are often also investigated.

The current routine use of electron microscopy (including microanalyses) is in the following expert examinations: - unknown samples (including powders from extortionate letters), - mineralogical, petrological and gemmological objects (mineral relics, soils, precious stones and their imitations, etc.), gunshot residues (GSR), - explosives, propellants and fulminating compounds, - post-blast residues (PBR), and other thermogenetic particles, [1] - fillers and additives of paper and plastics, - pigments and paint systems, including colour layers of artworks, - cosmetic and pharmaceutical products (surfaces and coating layers of tablets, granular composition, phase analysis), - morphological structures of textiles materials, - determination of sorts of damages to fibres (smelting, fibre fracture, tear/cut, fracture, etc.), expert examinations of biological materials - trichological material and its damage, shells of soil microorganisms, insect eggs to determine post mortem interval, etc.), - intersecting lines of writing and print tools (superposition - writing tool x print toner), - glass, - fragments of building materials, fractures of materials (determination of the character of the fracture area), - toolmark slipped impressions (forensic technical examinations, ballistics, etc.).

The possibilities of electron microscopy have been recently greatly enhanced by the use of dual systems with a focused ion beam. Probably the most widely used FIB technique in forensic science is the use of $\mathrm{Ga}+$ ions. These techniques allow the addition and removal of materials (molecular level), imaging in secondary ions and, optionally, performing ion microanalysis. For the time being, SEM/FIB technology has found applications in the following areas of forensic practice:

Analysis of superposition of toner/writing ink - finding superposition of strokes in the case of "reprints" on already signed documents is important to determine whether the signature was earlier on the document and then the other data were reprinted, or whether the signature followed the printing.

Holographic security features - are increasingly used to authorize prints, declare product originality, reliably detect unauthorized manipulation, etc. The objective of hologram analysis in forensic practice is to use detailed structural and other information to compare with the submitted comparative original samples and to detect counterfeits.

Thermogenetic particles - particles resulting from physical and chemical processes in which there is an immediate increase in temperature and pressure (post-blast residues, gunshot residues, particles of 
airbags, etc.). Thermogenetic particles have a specific chemical composition - according to primary genesis. The chemical components of the internal particle structure cannot in many cases be uniquely identified from the surface, and the phases that crystallize in the particle cavities are often also significant. The morphology of particle wall construction is also characteristic and therefore significant in many cases. All this information is important for the unambiguous inclusion of a particle into the appropriate generic category. [2]

Nanocomposites and nanoparticles - nanocomposites can be most commonly en-countered in variable colour pigments - protective elements (e.g. banknotes), automotive lacquers, consumer electronics, etc., and in functional glass layers (thermosetting, automotive glass, etc.). The aim of the analysis is to use detailed structural information to compare the presented comparative samples. Direct surface analysis again does not provide enough information for qualified comparisons.

Deformations in alloys - the aim is to visualize deformation changes of materials, their documentation and description, and possibly to determine the effects of deformations (fatigue fractures, pressure and temperature effects, etc.). Ion imaging allows, unlike electrons, good grain separation in metallic alloys and imaging of their micromorphology (similarly to an optical microscope), but with higher magnification and resolution. [3]

Another of the new areas of applications are the systems of automatic mineralogical analysis for the forensic analysis of pedological traces. In the field of soil exploration, SEM technologies are standardly used for analyses of mineral and anthropogenic phases, but automatic mineralogical analysis provides additional possibilities for obtaining accurate quantitative data. These systems are not self-sustaining, but they are complementary to existing analytical procedures. On the basis of the experiments, which were done and after the adjustment of the identification procedures, it is possible to analyze even untreated grains with natural surface, and not only embedded polished specimens. The area of the sample, which is not classified due to the topographic surface, is around $10 \%$, which is acceptable when comparing samples. Several series of random samples were taken from different locations of real samples and the ability to detect differences between individual sampling points was tested. It was found that the differences were traceable even between sampling points that were less than 20 meters apart.

The use of modern SEM techniques brings to forensic science new possibilities and use of evidence from traces that have been difficult to process or unusable in the past. Techniques are successfully used in a number of real cases where conventional techniques have been unable to provide a clear answer to investigators' questions [4].

\section{References:}

[1] Kotrlý M., Turková I.: Proc. SPIE 9486, (2015), p. 948614-1.

[2] Kotrlý M., Mareš B., Turková I., Beroun I.: Proc. SPIE 9823 (2016), p. 98230S-1.

[3] Kotrlý M., Microsc. Microanal. 23 (2017), p. 256.

[4] Microanalytical methods at ICP has been supported by the projects of the Ministry of the Interior of the Czech Republic: VI20152020004, VI20152020035, VI20172020050. 